\title{
A importância das competências do gestor da cadeia de suprimentos para a competitividade da organização
}

\author{
The importance of skills supply chain manager for the competitiveness of the \\ organization
}

\author{
Wanderson Fernandes Modesto de Oliveira1 \\ Domingos Fernandes Campos ${ }^{2}$ \\ Walid Abbas El-Aoua ${ }^{3}$
}

\begin{abstract}
Resumo
Este trabalho tem como objetivo analisar a importância das competências do gestor da cadeia de suprimentos para a competitividade das empresas. Realizou-se uma pesquisa qualitativa em literatura referente ao tema. Os resultados apontam a necessidade de as organizações desenvolverem vantagem competitiva em um contexto de cadeia de suprimentos, sendo as competências do gestor dessa cadeia atributos fundamentais à competitividade da empresa no atendimento ao pedido do cliente. Concluiu-se que, para a organização ser competitiva, ela necessita estar integrada em uma cadeia de suprimentos e o perfil do gestor daquela deve abranger competências técnicas (como conhecimento das necessidades dos clientes e de como atendê-las, conhecimento dos custos operacionais e financeiros, integração interna da empresa e com fornecedores e resolução dos problemas da cadeia de suprimentos), habilidades comportamentais (como trabalho em equipe, comunicação e foco no atendimento do cliente), e conhecimento da concorrência e dos processos centrais da cadeia de suprimentos.

Palavras-chave: Cadeia de suprimentos. Habilidades do gestor da cadeia de suprimentos. Competências do gestor da cadeia de suprimentos. Competitividade. Vantagem competitiva.

Abstract

This study aims to analyze the skills of the Manager of the supply chain for the competitiveness of enterprises. Qualitative research was undertaken in the literature concerning the topic. The results highlight the need for organisations to develop competitive advantage in a supply chain context, and the powers of the Manager of this chain are fundamental attributes to the company's competitiveness in meeting customer's request. We have come to the conclusions that the Organization be competitive it needs to be integrated into a supply chain and the same Manager profile should cover technical skills (knowledge of customer needs and how to meet them, financial and operational costs, the company's internal and integration with suppliers, solving the problems of the supply chain), behavioral skills (teamwork, communication, customer-focused), knowledge of competition and of the core supply chain processes.
\end{abstract}

Keywords: Supply chain. Supply chain manager skills. Supply chain competences. Competitiveness. Competitive advantage.

\section{Introdução}

No cenário contemporâneo de concorrência globalizada, as organizações necessitam identificar e desenvolver suas vantagens competitivas para poderem sobreviver, crescer e alcançar os resultados

\footnotetext{
Mestre em Administração - UNP. MBA em Gestão Estratégica de Negócios - UNI/RN. Graduação em Administração - UFRN. Brasil. Afiliação: Universidade Potiguar. Lattes: http://lattes.cnpq.br/3511939709684605 Email: wandersonf81@gmail.com

2 Doutor em Engenharia da Produção pela Universidad Politécnica de Madrid (1989). Concluiu seu Mestrado em Engenharia da Produção em 1979 na Universidade Federal de Santa Catarina. É Graduado em Engenharia Civil (1977) e em Psicologia (2004) pela Universidade Federal do Rio Grande do Norte. Coordena Pesquisas nas áreas de Gestão da Cadeia de Suprimentos e Gestão de Serviços. Brasil. Afiliação: Universidade Potiguar. Lattes: http://lattes.cnpq.br/7595790889667594 Email: domingoslogistica@gmail.com

3 Doutor em Administração pela Universidade Federal do Rio Grande do Norte. Pesquisas em: empreendedorismo, plano de negócio, qualidade de vida, estratégia competitiva e satisfação do cliente. Linha de Pesquisa: Gestão Estratégica de Pessoas. Brasil. Afiliação: Universidade Potiguar. Lattes: http://lattes.cnpq.br/9068744455564829 Email: walidbranco@gmail.com
} 
esperados, atendendo às demandas do mercado, lucrando e se fortalecendo estrategicamente em relação aos concorrentes (OLIVEIRA; EL-AOUAR; NÓBREGA, 2017; PORTER, 2004; PRAHALAD; HAMEL, 2005). Contudo a efetiva vantagem competitiva não é mais observada apenas nos aspectos da inovação, da tecnologia, dos custos ou da estratégia de uma organização isoladamente, mas, sim, no âmbito da cadeia de suprimentos (GUERREIRO; BIO; MENDEL, 2011), uma vez que a competição se dá entre cadeias de suprimentos contra cadeias de suprimentos, e não entre unidades individuais contra unidades individuais (LOPES et al., 2014; SCAVARDA; SCAVARDA, 2004).

Para Neutzling e da Silva (2016), um dos principais desafios para as empresas é desenvolver modelos de gestão capazes de conquistar e manter vantagens competitivas no mercado. Dentro dessa perspectiva, começam a emergir estudos que apontam para o papel do comportamento colaborativo interorganizacional como desenvolvedor de competitividade. Com isso, este estudo visa preencher uma lacuna observada na literatura que aborda a competitividade, a qual tem focado na inovação, na tecnologia, na melhoria dos processos ou nos custos (BATEMAN; SNELL, 2006; HITT; MILLER; COLELLA, 2007; PORTER, 2004; PRAHALAD; HAMEL, 2005; VAN DER HEIJDEN, 2009). Como a gestão da cadeia de suprimentos tem sido considerada uma variável estratégica para o desenvolvimento da competitividade das empresas (GUERREIRO; BIO; MENDEL, 2011), o objeto desta pesquisa foi analisar a importância das competências do gestor da cadeia de suprimentos para a competitividade das empresas. Justifica-se em função de seu aspecto exploratório no que se refere à associação entre os construtos "competências do gestor da cadeia de suprimentos" e "competitividade das empresas", identificando quais as competências consideradas essenciais para uma eficiente gestão da cadeia e seus impactos na competitividade.

Para as organizações, este trabalho contribui por entender os resultados do papel do gestor da cadeia de suprimentos na competitividade das empresas, podendo ser útil para que administradores procurem identificar e desenvolver as competências, habilidades e conhecimentos essenciais para que esses gestores levem as empresas a obterem os resultados planejados, contribuindo para o desenvolvimento das suas vantagens competitivas .

A descrição do perfil de competências do gestor da cadeia de suprimentos e das atividades que deve realizar, de suas metas de desempenho e dos benefícios extras que receberá com o atingimento dessas metas, poderá ser relevante para o incremento da competitividade das empresas. Para a ciência, este artigo tem potencial para o aperfeiçoamento teórico e empírico, tendo em vista se tratar de um tema ainda pouco explorado no Brasil.

Em relação às implicações gerenciais, este trabalho apresenta eficácia prática, pois seus achados justificam a adoção de estratégias de melhoria da eficiência da gestão da cadeia de suprimentos. Entre elas, a do desenvolvimento das competências e habilidades técnicas, do aprendizado de novos modelos de gestão e de incentivos aos gestores na obtenção dos resultados.

O presente estudo caracteriza-se como um ensaio teórico, de cunho reflexivo, pois não se limita a apresentar o que a literatura aborda sobre o tema, mas estabelece relações e sugere questionamentos (MENEGHETTI, 2011). Para tanto, nas seções seguintes, serão apresentadas as discussões teóricas sobre a competitividade, a globalização da competitividade, , a vantagem competitiva das empresas, a gestão da cadeia de suprimentos e a competitividade das empresas, e as competências do gestor da cadeia de suprimentos (GCS).

\section{Referencial teórico}

\subsection{A globalização da competitividade}

O conceito de globalização é diverso. Para Monteiro, Medina e Laureano (2001), a globalização pode ser entendida como a integração dos mercados em uma aldeia global, explorada pelas grandes corporações multinacionais, as quais competem nesses mercados e têm imposto uma nova forma de organização e novas estratégias competitivas às organizações. Ramalho e Sousa (2009) apontam que, na globalização dos mercados, os principais agentes econômicos são empresas internacionais que promovem a concentração do capital financeiro, de tecnologia para o desenvolvimento de produtos e processos, e dominam os sistemas 
de distribuição. Nesse aspecto, a concorrência global pode ser constatada no relatório da Revista Fortune publicado neste ano, o qual elenca as 500 maiores empresas do mundo, que geraram 27,6 trilhões de dólares em receitas e 1,5 trilhão em lucros em 2016. Essas companhias empregam 67 milhões de pessoas e estão presentes em 33 países, representando 56 tipos de indústrias. A Figura 1 apresenta os paísessede dessas 500 empresas. Observa-se que estão localizadas na União Europeia, na América do Norte, no Leste da Ásia e, em menor quantidade, no Novo Mundo, no Oriente Médio e no Brasil.

Figura 1 - Países sede das 500 maiores empresas do mundo

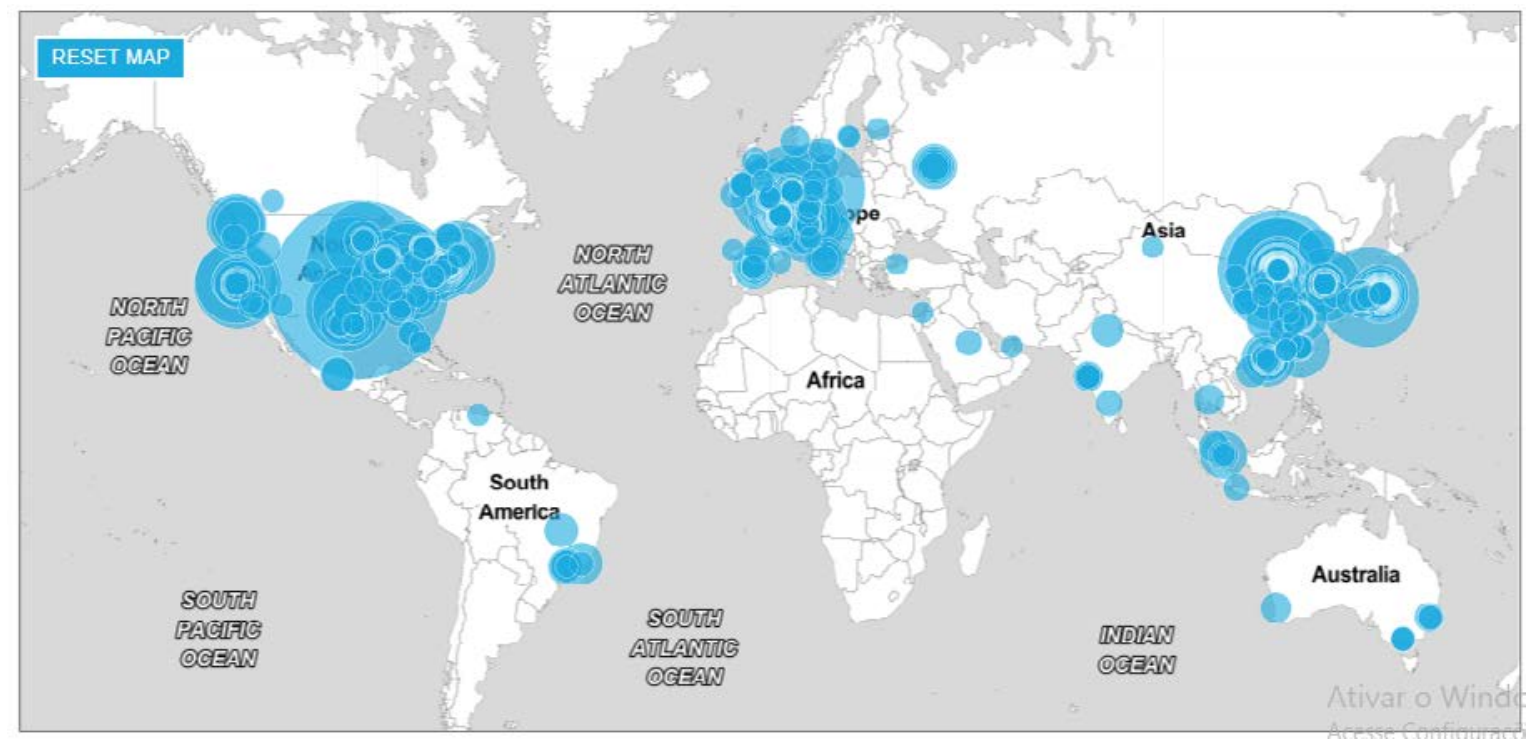

Fonte: Revista Fortune (2017)

Além das maiores empresas listadas pela Revista Fortune (2017), no contexto brasileiro, a concorrência também pode ser percebida pelo número de empresas que são abertas e estão atuantes no mercado. Dados do IBGE (2017) revelam que, entre 2007 e 2015, a taxa média de natividade de empresas foi de 10,8\%, algo em torno de 4.400 milhões de empresas sendo criadas a cada ano nesse período. Esse contínuo aumento de empresas (nacionais e internacionais) disputando um mesmo mercado elevou a concorrência local ao status de concorrência globalizada (MONTEIRO; MEDINA; LAUREANO, 2001).

Diante desse quadro das influências da globalização, tais como o acirramento da concorrência, a mudança nos hábitos de consumo, a maior rapidez nas inovações tecnológicas e a acentuação das alterações de valores, de crenças e de comportamentos sociais, é indispensável para a administração das organizações que elas sejam competitivas para enfrentar esses desafios (PRAHALAD; HAMEL, 2005; PORTER, 2004), sobreviverem e, sobretudo, crescerem, considerando que as empresas possuírem apenas as inovações tecnológicas não é mais condição suficiente (YEN-TSANG; CSILLAG; SIEGLER, 2012). Assim, diante dessa nova realidade competitiva, as organizações necessitam desenvolver a sua competitividade por meio de suas vantagens competitivas (FILHO; AMIGO, 2000).

\subsection{A competitividade e a vantagem competitiva das empresas}

O termo "vantagem competitiva" ganhou popularidade, principalmente no setor privado, por causa do trabalho de Michael Porter durante a década de 1980 do século passado (MOONEY, 2007). Para Porter (2004), a vantagem competitiva decorre da capacidade da empresa de criar um valor superior para seus compradores. As vantagens competitivas sustentáveis de uma organização são aquelas que os concorrentes não podem comprar, se apropriar ou copiar (WAGNER III; HOLLENBECK, 2009). Elas possibilitam que uma organização ofereça produtos e serviços melhores do que os dos concorrentes ou, pelo menos, que os faça iguais, mas a um custo estruturalmente menor (HITT; MILLER; COLELLA, 2007; VAN DER HEIJDEN, 2009).

Discorrendo sobre a relação entre competitividade e vantagem competitiva, Possas e Carvalho (1990) afirmam que: 
A competitividade é 'o poder de definir (formular e implantar) estratégias de valorização do capital, desde que baseado em aspectos econômicos e não institucionais' [...]. Quer dizer, deve estar respaldado na posse de vantagens competitivas com maior ou menor eficácia em face das dos demais concorrentes, tanto no momento presente quanto no que se espera que seja o futuro próximo. (POSSAS; CARVALHO, 1990, P. 53 apud POSSAS, 1999, p. 173-174)

Porter (2004) desenvolveu a teoria de que existem cinco forças competitivas determinantes estruturais da intensidade da concorrência: 1) A ameaça da entrada de novos concorrentes no mercado; 2) A ameaça da substituição dos produtos dos concorrentes; 3) O poder de negociação dos compradores; 4) O poder de negociação dos fornecedores e 5) A rivalidade entre os atuais concorrentes. Ele, então, estabelece três abordagens que, como estratégia para superar a concorrência, uma organização necessita adotar para ser competitiva: a) A liderança do custo total do produto (ela deve ter o custo total menor do que os concorrentes); b) A diferenciação do seu produto ou serviço em relação aos concorrentes, de modo que ele seja considerado único pelo mercado; c) O enfoque (quando é enfocado um determinado grupo de comprador ou um segmento da linha de produtos ou um mercado geográfico específico).

Randons et al. (2012) assinalam que o ambiente atual se caracteriza como complexo, incerto e mutável, apresentando novos desafios para empresas, e que, por isso, elas demandam novas perspectivas sobre a competitividade. Esses autores entendem que uma constante revisão de suas posições competitivas e uma adaptação ao mercado são exigidas das empresas. Nesse processo de revisão, ao longo dos anos, as empresas têm alterado suas estratégias a fim de se tornarem mais competitivas no mercado (RANDONS et al., 2012).

Por causa disso, diversas abordagens objetivando estender a competitividade das organizações têm sido estudadas no decorrer dos anos, tais como a inovação (PAYÉS; SILVA; TEIXEIRA, 2008), a sustentabilidade (STARIK; KANASHIRO, 2013), responsabilidade social (BREITBARTH; HARRIS; AITKEN, 2009), as redes de organizações (SCHREIBER; VILELA JÚNIOR; BANDEIRA, 2007) e a gestão da cadeia de suprimentos (GUERREIRO; BIO; MENDEL, 2011).

Por sua vez, Certo e Peter (2010) defendem que a criação do futuro para a organização é possível por meio da coordenação de todos os seus recursos, com a finalidade de obter a vantagem competitiva, usando para isso uma estrutura de planejamento cuidadosamente elaborada, flexibilizando os processos de execução desse planejamento e criando um clima de apoio interno. De acordo com Oliveira et al. (2015), o capital humano (ou o capital intelectual) eficiente é o que operacionaliza as vantagens competitivas de Porter (2004), sendo, então, o principal diferencial entre as organizações bem-sucedidas (ORLICKAS, 2011), inclusive no contexto da gestão da cadeia de suprimentos (BRAVO; MORENO; LLORENS-MONTES, 2016).

\subsection{A competitividade e a gestão da cadeia de suprimentos}

A gestão da cadeia de suprimentos tem sido considerada um dos principais fatores condicionantes da competitividade das organizações (GUERREIRO; BIO; MENDEL, 2011; MENTZER et al., 2001; PRAJOGO; SOHAL, 2013). No ambiente globalizado, as empresas constataram que a concorrência já não depende apenas das capacidades das empresas individuais, mas da capacidade de uma cadeia de suprimentos totalmente integrada entre seus membros (TAN; LYMAN; WISNER, 2002; MIN; ZHOU; 2002; PRAJOGO; SOHAL, 2013; SCAVARDA; SCAVARDA, 2004). A cooperação entre os membros de uma cadeia de suprimento favorece para que determinada empresa alcance vantagem competitiva com suas empresas parceiras (fornecedores e clientes) em relação aos concorrentes (CHANG; CHIANG; PAI, 2012; FLYNN; HUO; ZHAO, 2010). A cadeia de suprimentos abrange todos os estágios e processos relacionados, direta e indiretamente, ao atendimento eficiente do pedido do cliente (BALLOU, 2006; CHOPRA; MEINDL, 2003; SAUBER; MCSURELY; TUMMALA, 2008).

Stock e Boyer (2009) elaboraram a seguinte definição de gestão da cadeia de suprimentos:

[É] A gestão de uma rede de relacionamentos dentro de uma empresa e entre organizações interdependentes e unidades de negócios que consistem em fornecedores de materiais, compras, instalações de produção, logística, marketing e sistemas relacionados 
que facilitam o fluxo bidirecional de materiais, serviços, finanças e informação de o produtor original para cliente final com os benefícios da adição de valor, maximizando de rentabilidade através de ganhos de eficiência e alcance da satisfação do cliente. (STOCK; BOYER, 2009, p. 706, tradução nossa)

Rossetti e Dooley (2010) entendem a existência de oito funções relacionadas à gestão da cadeia de suprimentos: (1) Gestão de suprimentos; (2) Consultor de operações; (3) Gestor de operações de serviços; (4) Gestor de planejamento de compras; (5) Gestor de informação em cadeia de suprimentos; (6) Gestor de integração logística; (7) Gestor de logística externa e (8) Gestor de manufatura.

Por isso, os profissionais envolvidos com a gestão da cadeia de suprimentos necessitam possuir o perfil profissional adequado, por meio de um conjunto de conhecimentos, competências e habilidades técnicas e comportamentais, para realizar uma eficiente gestão da cadeia, de forma que seus membros atinjam os resultados esperados (ABREU; ALCÂNTARA, 2014b; AB TALIB; ABDUL HAMID, 2014).

Ribeiro e Neto (2017) ressaltam que, com a globalização, produtos, processos, inovações, estratégias e tecnologias são facilmente copiáveis e adaptáveis entre as organizações, mas, por outro lado, as competências de um gestor, que o leva a obter os resultados planejados pela organização, é a vantagem competitiva mais sustentável no tempo, e constitui a fonte de criatividade e inovação constante das empresas.

\section{As competências do gestor da cadeia de suprimentos (GCS)}

O conceito de competência é amplo. Para Montezano, Abbad e Freitas (2016, p. 3), por exemplo, a competência é a "expressão do conjunto de conhecimentos, habilidades e atitudes no contexto de trabalho, por meio de comportamentos observáveis que geram desempenhos e resultados bem-sucedidos". Já para Fleury e Fleury (2001, p. 4), a competência é "um saber agir responsável e reconhecido, que implica mobilizar, integrar, transferir conhecimentos, recursos e habilidades, que agreguem valor econômico à organização e valor social ao indivíduo". Alinhado com essas perspectivas, Le Boterf (1999) assinala que a competência da pessoa é consequência da aplicação conjunta das três dimensões da competência: conhecimentos, habilidades e atitudes.

No contexto da competência do gestor da cadeia de suprimentos, Jacomino, Bánkuti e Vieira (2018) entendem que a gestão da cadeia de suprimentos é qualificada pela coordenação de atividades e processos de negócios, por meio de relações intra e interorganizacionais, envolvendo os fornecedores e os clientes pertencentes aos diversos níveis da cadeia de suprimentos. Esse gestor poderá ser um empregado da empresa que fornece ao cliente final, atuando como um elo de coordenação entre as diversas empresas que fazem parte da cadeia de suprimentos (GAMMELGAARD; LARSON, 2001). A gestão de pessoas nos aspectos comportamentais, técnicos e profissionais tem sido o objeto de grandes pesquisas nos estudos relacionados à cadeia de suprimento em razão das competências humanas serem relevantes para o sucesso de sua gestão (GUNASEKARAN; PATEL; MCGAUGHEY, 2004). Snell e Bohlander (2013) advogam que as empresas "competem por meio das pessoas", significando que o sucesso depende, cada vez mais, da capacidade empresarial de gerenciar o capital humano, o qual se relaciona com o quadro pessoal dentro de uma organização e que inclui o conhecimento tácito, a habilidade, a atitude e a experiência dos empregados (OLIVEIRA; EL-AOUAR; LEONE, 2017).

Nesse contexto, após examinarem 300 empresas europeias para identificar os fatores mais importantes para a reconfiguração da cadeia de suprimento, objetivando maximizar sua eficácia, Van Hoek, Chatham e Wilding (2002) constataram que o fator mais importante foi a cadeia dispor de profissionais qualificados, mais do que a infraestrutura física, a qualidade e a rapidez da informação e dos processos de comunicação.

Giunipero, Handfield e Eltantawy (2006) listam cinco habilidades que são essenciais para que os gestores da cadeia de suprimentos atuem com eficiência: (1) Habilidades para montar equipes: liderança, tomada de decisão, influência e comprometimento; (2) Habilidades de planejamento estratégico: elaboração de projeto, definição de metas e de execução; (3) Habilidades de comunicação: apresentação, falar em público, ouvir e escrever; (4) Habilidades técnicas: pesquisar e analisar na web; e (5) Habilidades financeiras mais amplas: contabilidade de custos e realização de cases de negócio. 
Closs e Mollenkopf (2004) elaboraram um quadro contendo as seis competências críticas para a logística e para a gestão da cadeia de suprimentos do século XXI, que podem ser agrupadas em processos: operacional, de planejamento e comportamental. O processo operacional envolve a integração do cliente, interna e com o fornecedor (tanto para bens como para serviços). O processo de planejamento inclui competências relacionadas à tecnologia e à integração de planejamento com medição. O processo comportamental se refere à integração do relacionamento com clientes e fornecedores.

Em uma pesquisa realizada entre profissionais da cadeia de suprimentos na Austrália, Prajogo e Sohal (2013) listaram as competências que os respondentes identificaram como as mais importantes para a gestão eficiente da cadeia de suprimentos. A capacidade de trabalhar de forma eficaz com indivíduos e grupos/equipe (transculturalmente, intra e interorganização) e a capacidade de gerenciar relacionamentos em diversos contextos (transculturalmente, intra e interorganização) foram as competências consideradas mais importantes, obtendo as médias de 4,39 e 4,23, respectivamente. A capacidade de gerenciar riscos na cadeia de suprimentos e os problemas associados obteve a média de 4,11 , ficando em terceiro lugar numa escala decrescente de valor. A capacidade de fazer uso de técnicas numéricas para a tomada de decisão (por exemplo, forecasting e scheduling), e as habilidades de gerenciamento de projetos e capacidade de liderar grandes projetos obtiveram as médias de 4,10 e 4,09, respectivamente.

Por fim, obtiveram a mesma média, de 4,0, a capacidade de aplicar melhorias contínuas, o conceito de foco no cliente e a "capacidade de gerir mudanças dentro do contexto local. Baseando-se nos resultados dessa pesquisa, Prajogo e Sohal (2013) consideram que comunicação e trabalho em equipe são as competências mais importantes do gestor para o sucesso da integração da cadeia de suprimentos.

\section{Metodologia}

Os procedimentos metodológicos adotados seguem os padrões da pesquisa teórica, a qual requer que seja construída de forma sistemática e rigorosa, condição indispensável para credibilidade dos resultados assinalados no trabalho, bem como para a formação de conhecimento em uma base sólida a partir dele (TRANFIELD; DENYER; SMART, 2003).

Assim, para este ensaio teórico, optou-se por uma pesquisa qualitativa, de abordagem exploratória, pois pretende-se examinar um tema pouco estudado, que auxilia nas definições do problema da pesquisa, bem como justifica a necessidade da realização do estudo (SAMPIERI; COLLADO, LÚCIO, 2013; SNYDER, 2012) e demonstra a verdade não por meio de dados quantitativos ou numéricos (como ocorre na pesquisa quantitativa), mas na forma de argumentação estruturada, lógica e bem fundamentada das ideias (MICHEL, 2015).

A coleta dos dados foi secundária, uma vez que os artigos foram extraídos das bases de pesquisa que são referência no mundo acadêmico (HARZING; ALAKANGAS, 2015), apresentadas no Quadro 1, visando à realização de estudo bibliométrico e bibliográfico, pois permitem examinar uma variedade de assuntos para ajudar a entender, organizar e explorar o que já foi feito ou pesquisado a partir das fontes bibliográficas (FERREIRA, 2011). A pesquisa nas fontes de dados foi realizada com os parâmetros listado no Quadro 1, os quais foram utilizados para ambos os estudos.

Quadro 1 - Parâmetros de pesquisa

\begin{tabular}{|c|l|l|l|}
\hline $\begin{array}{c}\text { Base de } \\
\text { pesquisa }\end{array}$ & \multicolumn{1}{|c|}{ Grande área } & \multicolumn{1}{|c|}{$\begin{array}{c}\text { Linha de pesquisa } \\
\text { pesquisado }\end{array}$} \\
\hline Ebsco Host & $\begin{array}{l}\text { Bussiness; Economics } \\
\text { Social Sciences \& } \\
\text { Humanities }\end{array}$ & $\begin{array}{l}\text { Accounting \& Finance; Economics; HR \& } \\
\text { Organizational Behaviour; } \\
\text { Information \& Knowledge; Management; } \\
\text { Management Science \& Operations; } \\
\text { Marketing; Property Management \& Built } \\
\text { Environment; Public Policy \& Environmental } \\
\text { Management; Strategy }\end{array}$ & $\begin{array}{l}\text { Journals, books, } \\
\text { review, conference } \\
\text { paper and article }\end{array}$ \\
\hline
\end{tabular}




\begin{tabular}{|l|l|l|l|}
\hline Emerald & $\begin{array}{l}\text { Bussiness; Economics } \\
\text { Social Sciences \& } \\
\text { Humanities }\end{array}$ & $\begin{array}{l}\text { Accounting \& Finance; Economics; } \\
\text { Information \& Knowledge; Management; } \\
\text { Management Science \& Operations; } \\
\text { Marketing; Management \& Built Environment; } \\
\text { Strategy }\end{array}$ & $\begin{array}{l}\text { Journals, books, } \\
\text { review and article }\end{array}$ \\
\hline ProQuest & Social Sciences & $\begin{array}{l}\text { Applied Social Sciences Index \& Abstracts } \\
\text { (ASSIA) }\end{array}$ & $\begin{array}{l}\text { Journals, books, } \\
\text { review and article }\end{array}$ \\
\hline Science Direct & $\begin{array}{l}\text { Bussiness; Economics } \\
\text { Social Sciences \& } \\
\text { Humanities }\end{array}$ & $\begin{array}{l}\text { Business, Management and Account; Social } \\
\text { Sciences; Economics, Econometrics and } \\
\text { Finance; Decision Sciences; Environmental } \\
\text { Science }\end{array}$ & $\begin{array}{l}\text { Journals, books, } \\
\text { review and article }\end{array}$ \\
\hline Scopus & $\begin{array}{l}\text { Suciness, Management and Account; Social } \\
\text { Sciences; Economics, Econometrics and } \\
\text { Sciences\&Humanities } \\
\text { Finance; Decision Sciences; Environmental } \\
\text { Science }\end{array}$ & $\begin{array}{l}\text { Journals, books, } \\
\text { review and article }\end{array}$ \\
\hline
\end{tabular}

Fonte: Elaboração dos autores.

\subsection{Procedimentos de análise}

Para a análise dos dados, foi utilizada a técnica de análise documental, por meio da qual os dados foram tabulados e analisados de acordo com o objetivo deste estudo (PIMENTEL, 2001). A análise documental se mostra como a melhor escolha, uma vez que a sua finalidade é esboçar uma visão geral do que já foi publicado, e não necessariamente produzir novos conhecimentos (SILVA; FECHIO; PEPECE, 2014).

Primeiramente, foram pesquisados artigos publicados no período de 2000 a 2016, utilizando-se, para tanto, das palavras-chave relacionadas ao tema deste trabalho, sendo elas: Supply chain, supply chain manager skills, supply chain manager competences, competitiveness e competitive advantage, Supply chain manager competences and Competitivenes. Para efetivar a triagem dos artigos, selecionou-se nas fontes de pesquisa que as publicações fossem organizadas por ordem decrescente de relevância.

Em uma segunda etapa, foi realizada a triagem dos artigos para a pesquisa bibliográfica, os quais foram selecionados pelos critérios de: (1) maior afluência de conteúdo para com este estudo; (2) terem sido altamente citados; (3) serem de relevância para a temática; e, (4) associarem as palavraschave: Supply chain manager skills - Competitiveness, Supply chain manager skills - Competitive advantage e Supply chain manager competences - Competitive advantage. Nesta etapa, 231 (17,5\% do total) artigos foram pré-selecionados e estão quantificados na Tabela 3. Por fim, foram lidos os respectivos títulos, resumos e palavraschave desses 231 artigos, filtrando aqueles que tinham maior aderência com o objetivo deste trabalho, maior número de citações, e o fator de impacto (SJR) e $\mathrm{H}$-Index considerados bons, os quais estão listados no Quadro 2.

Quadro 2 - Artigos selecionados

\begin{tabular}{|l|l|l|l|l|l|}
\hline \multicolumn{1}{|c|}{ Autor(es) } & \multicolumn{1}{|c|}{ Título } & \multicolumn{1}{c|}{ Periódico } & $\begin{array}{l}\text { Número de } \\
\text { citações }^{1}\end{array}$ & $\begin{array}{c}\text { Fator de } \\
\text { impacto }^{2}\end{array}$ & $\begin{array}{c}\text { H } \\
\text { Index }^{3}\end{array}$ \\
\hline MOONEY, A. & $\begin{array}{l}\text { Core competence, } \\
\text { distinctive competence, and } \\
\text { competitive advantage: what } \\
\text { is the difference? }\end{array}$ & $\begin{array}{l}\text { Journal of Education } \\
\text { for Business }\end{array}$ & 100 & --- & --- \\
\hline $\begin{array}{l}\text { STARIK, M.; } \\
\text { KANASHIRO, P. }\end{array}$ & $\begin{array}{l}\text { Toward a Theory of } \\
\text { Sustainability Management: } \\
\text { Uncovering and Integrating } \\
\text { the Nearly Obvious. }\end{array}$ & $\begin{array}{l}\text { Organization \& } \\
\text { Environment }\end{array}$ & 111 & 0,76 & 38 \\
\hline $\begin{array}{l}\text { BREITBARTH, } \\
\text { T.; HARRIS, P.; } \\
\text { AITKEN, R. }\end{array}$ & $\begin{array}{l}\text { Corporate social } \\
\text { responsibility in the } \\
\text { European Union: a new } \\
\text { trade barrier? }\end{array}$ & $\begin{array}{l}\text { Journal of Public } \\
\text { Affairs }\end{array}$ & 39 & 0,124 & 13 \\
\hline
\end{tabular}




\begin{tabular}{|c|c|c|c|c|c|}
\hline $\begin{array}{l}\text { MENTZER, J. T. } \\
\text { et al. }\end{array}$ & $\begin{array}{l}\text { Defining supply chain } \\
\text { management. }\end{array}$ & $\begin{array}{l}\text { Journal of Business } \\
\text { Logistics }\end{array}$ & 4.332 & ----- & 21 \\
\hline $\begin{array}{l}\text { PRAJOGO, D.; } \\
\text { SOHAL, A. }\end{array}$ & Supply chain professionals. & $\begin{array}{l}\text { International Journal } \\
\text { of Operations } \\
\text { \& Production } \\
\text { Management }\end{array}$ & 38 & 1,36 & 104 \\
\hline $\begin{array}{l}\text { TAN, K.; LYMAN, } \\
\text { S. B.; WISNER, } \\
\text { J. D. }\end{array}$ & $\begin{array}{l}\text { Supply chain management: } \\
\text { a strategic perspective }\end{array}$ & $\begin{array}{l}\text { International Journal } \\
\text { of Operations } \\
\text { \& Production } \\
\text { Management }\end{array}$ & 630 & 1,026 & 104 \\
\hline $\begin{array}{l}\text { MIN, H.; ZHOU, } \\
\text { G. }\end{array}$ & $\begin{array}{l}\text { Supply chain modeling: } \\
\text { past, present and future. }\end{array}$ & $\begin{array}{l}\text { Computers } \\
\text { \& Industrial } \\
\text { Engineering }\end{array}$ & 1.128 & 0,477 & 98 \\
\hline $\begin{array}{l}\text { CHANG, Cheng- } \\
\text { Wen; CHIANG, D. } \\
\text { M; PAI, Fan-Yun. }\end{array}$ & $\begin{array}{l}\text { Cooperative strategy in } \\
\text { supply chain networks. }\end{array}$ & $\begin{array}{l}\text { Industrial Marketing } \\
\text { Management }\end{array}$ & 57 & 1,504 & 100 \\
\hline $\begin{array}{l}\text { FLYNN, B. B.; } \\
\text { HUO, B.; ZHAO, } \\
\text { X. }\end{array}$ & $\begin{array}{l}\text { The impact of supply chain } \\
\text { integration on performance: } \\
\text { a contingency and } \\
\text { configuration approach. }\end{array}$ & $\begin{array}{l}\text { Journal of } \\
\text { Operations } \\
\text { Management }\end{array}$ & 1.376 & 4,902 & 149 \\
\hline $\begin{array}{l}\text { VAN HOEK, R.I.; } \\
\text { CHATHAM, R; } \\
\text { WILDING, R }\end{array}$ & $\begin{array}{l}\text { Managers in supply chain } \\
\text { management: the critical } \\
\text { dimension. }\end{array}$ & $\begin{array}{l}\text { Supply Chain } \\
\text { Management: An } \\
\text { International Journal }\end{array}$ & 111 & 0,711 & 84 \\
\hline $\begin{array}{l}\text { GUNASEKARAN, } \\
\text { A.; PATEL, C.; } \\
\text { MCGAUGHEY, } \\
\text { R. E. }\end{array}$ & $\begin{array}{l}\text { A framework for supply } \\
\text { chain performance } \\
\text { measurement }\end{array}$ & $\begin{array}{l}\text { International Journal } \\
\text { of Production } \\
\text { Economics }\end{array}$ & 1.992 & 1,335 & 131 \\
\hline $\begin{array}{l}\text { GIUNIPERO, L.C., } \\
\text { HANDFIELD, } \\
\text { R.B.; } \\
\text { ELTANTAWY, R. }\end{array}$ & $\begin{array}{l}\text { Supply management's } \\
\text { evolution: key skill sets for } \\
\text { the supply manager of the } \\
\text { future. }\end{array}$ & $\begin{array}{l}\text { International Journal } \\
\text { of Operations } \\
\text { \& Production } \\
\text { Management }\end{array}$ & 206 & 0,844 & 104 \\
\hline $\begin{array}{l}\text { CLOSS, D.J.; } \\
\text { MOLLENKOPF, } \\
\text { D. A. }\end{array}$ & $\begin{array}{l}\text { A global supply chain } \\
\text { framework. }\end{array}$ & $\begin{array}{l}\text { Industrial Marketing } \\
\text { Management }\end{array}$ & 126 & 0,76 & 100 \\
\hline $\begin{array}{l}\text { BRAVO, M. I. R.; } \\
\text { MORENO, A. } \\
\text { R.; LLORENS- } \\
\text { MONTES, F. J. }\end{array}$ & $\begin{array}{l}\text { Supply network-enabled } \\
\text { innovations. An analysis } \\
\text { based on dependence } \\
\text { and complementarity of } \\
\text { capabilities. }\end{array}$ & $\begin{array}{l}\text { Supply Chain } \\
\text { Management: An } \\
\text { International Journal }\end{array}$ & 3 & 1,864 & 84 \\
\hline $\begin{array}{l}\text { ROSSETTI, C. L.; } \\
\text { DOOLEY, K. J. }\end{array}$ & $\begin{array}{l}\text { Job types in the supply } \\
\text { chain management } \\
\text { profession. }\end{array}$ & $\begin{array}{l}\text { Journal of Supply } \\
\text { Chain Management }\end{array}$ & 32 & 0,722 & 47 \\
\hline
\end{tabular}

Fonte: Elaboração dos autores.

${ }^{1}$ Dados coletados no Google Scholar

2, 3 Dados coletados no http://www.scimagojr.com

Para a fase bibliométrica, a contabilização dos trabalhos foi feita no período de 7 a 10 de setembro de 2016 e, para a bibliográfica, no período de outubro a novembro de 2016.

\section{Resultados e discussões}

Nas bases pesquisadas, no período de 2000 a 2016, dentre as palavras-chave, supply chain foi a que mais se destacou (representando $48,02 \%$ do total), seguida por competitiveness $(21,02 \%)$ e competitive 
advantage (19,43\%). Já no somatório das cinco palavras-chave, foi na base de pesquisa Scopus em que foi encontrado um número mais expressivos de artigos (40.358), seguida pela ProQuest (com 19.057 artigos). Tais informações podem ser observadas na Tabela 2.

Esse volume de publicações na Scopus e na ProQuest deu-se em função de serem, entre as cinco bases pesquisadas, as que possuem, respectivamente, em sua base de dados, cerca de 64 milhões (o que inclui mais 21.548 títulos) e mais de 4 milhões de registros (incluindo mais de 4.560 títulos), conforme informações coletadas nos respectivos sites.

Tabela 2 - Palavraschave nas bases de pesquisa

\begin{tabular}{|c|c|c|c|c|c|c|c|}
\hline Palavraschave & $\begin{array}{c}\text { Ebsco } \\
\text { Host }\end{array}$ & Emerald & ProQuest & Scopus & $\begin{array}{l}\text { Science } \\
\text { Direct }\end{array}$ & Total & $\%$ \\
\hline Supply chain & 5.781 & 3.051 & 20 & 27.578 & 5.915 & 42.345 & 48,02 \\
\hline $\begin{array}{l}\text { Supply chain manager } \\
\text { skills }\end{array}$ & 3.237 & 3 & 4.049 & 100 & 4 & 7.393 & 8,38 \\
\hline $\begin{array}{l}\text { Supply chain manager } \\
\text { competences }\end{array}$ & 4 & 18 & 2.606 & 134 & 10 & 2.772 & 3,14 \\
\hline Competitiveness & 4.813 & 1.939 & 1.654 & 9.234 & 893 & 18.533 & 21,02 \\
\hline Competitive advantage & 1.905 & 806 & 10.728 & 3.312 & 385 & 17.136 & 19,43 \\
\hline Total & 15.740 & 5.817 & 19.057 & 40.358 & 7.207 & 88.179 & 100,00 \\
\hline
\end{tabular}

Fonte: Elaboração dos autores.

Das associações envolvendo as palavraschave, supply chain and competitive advantage estão presentes em $55,73 \%$ dos artigos publicados nas bases de pesquisa consultadas, sendo que supply chain and competitiveness estiveram relacionadas em $26,73 \%$ dos artigos (Tabela 3 ). Esses números sugerem que, para os autores de 734 artigos, há uma relação entre cadeia de suprimentos e vantagem competitiva, assim como, para os autores de 352 artigos, existe uma relação entre "cadeia de suprimentos" com "competitividade" e "competências do gestor da cadeia de suprimentos" com "competitividade". Essas relações serão discorridas nas seções a seguir.

Tabela 3 - Associação de palavraschave

\begin{tabular}{|c|c|c|c|c|c|c|c|}
\hline Palavraschave & $\begin{array}{c}\text { Ebsco } \\
\text { Host }\end{array}$ & Emerald & ProQuest & Scopus & $\begin{array}{l}\text { Science } \\
\text { Direct }\end{array}$ & Total & $\%$ \\
\hline $\begin{array}{l}\text { Supply chain and } \\
\text { Competitiveness }\end{array}$ & 11 & 0 & 0 & 341 & 0 & 352 & $26,73 \%$ \\
\hline $\begin{array}{l}\text { Supply chain and } \\
\text { competitive advantage }\end{array}$ & 4 & 42 & 0 & 687 & 1 & 734 & $55,73 \%$ \\
\hline $\begin{array}{l}\text { Supply chain manager skills } \\
\text { and Competitiveness }\end{array}$ & 83 & 0 & 0 & 1 & 0 & 84 & $6,38 \%$ \\
\hline $\begin{array}{l}\text { Supply chain manager skills } \\
\text { and competitive advantage }\end{array}$ & 18 & 0 & 0 & 4 & 0 & 22 & $1,67 \%$ \\
\hline $\begin{array}{l}\text { Supply chain manager } \\
\text { competences and } \\
\text { Competitiveness }\end{array}$ & 61 & 0 & 0 & 0 & 0 & 61 & $4,63 \%$ \\
\hline $\begin{array}{l}\text { Supply chain manager } \\
\text { competences and } \\
\text { competitive advantage }\end{array}$ & 14 & 0 & 0 & 50 & 0 & 64 & $4,86 \%$ \\
\hline Total & 191 & 42 & 0 & 1.083 & 1 & 1.317 & $100,00 \%$ \\
\hline
\end{tabular}

Fonte: Elaboração dos autores. 


\subsection{A necessidade de a empresa ser competitiva}

Ficou evidenciada a globalização da concorrência nos dados referentes a 2016, apresentados na Introdução, os quais revelam que as 500 maiores empresas do mundo estão sediadas em 33 países, concentrando-se na União Europeia, na América do Norte, no Leste da Ásia e, uma menor quantidade, no Novo Mundo, no Oriente Médio e no Brasil, e que geraram 27,6 trilhões de dólares em receitas. Além dessas empresas concorrentes, especificamente no cenário brasileiro, entre 2007 e 2015, a taxa média de natividade de empresas foi de 10,8\%, algo em torno de 4.400 milhões de empresas sendo criadas a cada ano no período.

Para enfrentar os desafios da concorrência, as empresas necessitam desenvolver suas vantagens competitivas. Isso foi observado na pesquisa bibliométrica realizada nas bases de pesquisa: 35.669 dos artigos publicados $(40,4 \%)$ tratam de temas como vantagem competitiva e competitividade. Esses dados se alinham com o referencial teórico estudado, pois, de acordo com Randons et al. (2012), o cenário atual é complexo, incerto e mutável, apresenta desafios que mudam constantemente, por isso requer das organizações frequentes adaptações às novas demandas da competitividade. Corroborando com essa perspectiva, Bateman e Snell (2006), Hitt, Miller e Colella (2007) e Porter (2004) propõem que as organizações necessitam buscar a vantagem competitiva para agregar valor aos seus clientes e, ao mesmo tempo, crescerem e lucrarem, sendo por meio do capital humano eficiente que as vantagens competitivas são desenvolvidas pelas organizações, tornando-se, então, o principal diferencial entre as bem-sucedidas (ORLICKAS, 2011).

O fator humano eficiente, considerado como verdadeira vantagem competitiva, ao invés da tecnologia, dos produtos, dos processos e estratégias, pois são facilmente copiados pela concorrência, é a abordagem inovadora no campo da vantagem competitiva (RIBEIRO; NETO, 2017). Certo e Peter (2010) foram autores que ressaltaram que a vantagem competitiva se realiza, efetivamente, quando todos os membros da empresa coordenam seus esforços para criá-la e desenvolvê-la.

A vantagem competitiva não é mais entendida como sendo presente apenas na liderança do custo total do produto, na diferenciação do seu produto ou serviço em relação aos concorrentes, no enfoque (PORTER, 2004) na inovação (PAYÉS; SILVA; TEIXEIRA, 2008), na sustentabilidade (STARIK; KANASHIRO, 2013), na responsabilidade social (BREITBARTH; HARRIS; AITKEN, 2009) ou nas redes de organizações (SCHREIBER; VILELA JÚNIOR; BANDEIRA, 2007). Mas, principalmente, ela está presente em uma eficiente gestão da cadeia de suprimentos (GUERREIRO; BIO; MENDEL, 2011). Essa tese é defendida também por Bravo, Moreno e Llorens-Montes (2016). Para eles, uma organização é bem-sucedida e competitiva no contexto de cadeia de suprimentos.

\subsection{A cadeia de suprimentos e a vantagem competitiva das organizações}

$\mathrm{Na}$ associação das palavraschave, $82,4 \%$ do total dos artigos publicados nas bases de pesquisa apresentaram correlação entre supply chain and competitive advantage e supply chain and competitiveness. Essa relação confirma o que está proposto pelos artigos pesquisados, para os quais: (i) o contexto que abrange a cadeia de suprimentos envolve todos os estágios e processos relacionados, direta e indiretamente, ao atendimento eficiente do pedido do cliente, ou seja, essa cadeia não se limita apenas ao aspecto da logística do fornecedor entregar o produto à empresa para que esta o transforme e/ou venda ao cliente (BALLOU, 2006; CHOPRA; MEINDL, 2003; SAUBER; MCSURELY; TUMMALA, 2008); (ii) a cooperação entre os membros de uma cadeia de suprimento favorece para que determinada empresa alcance vantagem competitiva com suas empresas parceiras (fornecedores e clientes) em relação aos concorrentes que procuram competir isoladamente (CHANG; CHIANG; PAI, 2012; FLYNN; HUO; ZHAO, 2010); (iii) a plena competitividade de uma organização é obtida em um contexto de cadeia de suprimentos, e não mais em função das vantagens competitivas individuais da própria empresa (TAN; LYMAN; WISNER, 2002; MIN; ZHOU, 2002; PRAJOGO; SOHAL, 2013; SCAVARDA; SCAVARDA, 2004).

Realizar uma gestão eficiente da cadeia de suprimentos é que favorecerá para que a organização desenvolva a vantagem competitiva em relação aos seus concorrentes, contribuindo, também, no atendendo 
das demandas do mercado em condições superiores à concorrência (CHANG; CHIANG; PAI, 2012; FLYNN; HUO; ZHAO, 2010). Por outro lado, percebeu-se que a literatura analisada não apresentou modelos que delineassem os componentes e processos que constituem uma cadeia de suprimentos, descrevendo, no máximo, a grosso modo, as figuras do: fornecedor $\rightarrow$ empresa $\rightarrow$ cliente.

\subsection{As competências do gestor da cadeia de suprimentos e a competitividade das empresas}

Na literatura pesquisada, a qualidade do capital humano em toda a cadeia de suprimentos é tida como substancial para que o pedido do cliente chegue à destinação final com qualidade, rapidez e economicidade, resultando na satisfação dele e na geração do lucro para a empresa que atendeu ao pedido. Isso posto, constatou-se que não é mais apenas o fato da empresa dispor de inovações tecnológicas, ou de produtos inovadores, de custos competitivos e de um planejamento estratégico eficaz que lhe confere vantagem competitiva, mas ter em seu quadro de pessoal o capital humano eficiente (GUNASEKARAN; PATEL; MCGAUGHEY, 2004; VAN HOEK; CHATHAM; WILDING, 2002).

Desse modo, levando em consideração o diferencial que um empregado eficiente produz para a empresa, Abreu e Alcântara (2014b) e AbTalib e Abdul Hamid (2014) defendem que os profissionais envolvidos com a gestão da cadeia de suprimentos necessitam ter um conjunto de conhecimentos, competências, habilidades técnicas e comportamentais para que a organização tenha vantagem competitiva diante da concorrência e obtenha os resultados esperados no atendimento dos clientes.

De acordo com a Tabela 3, a predominância de artigos que fazem associações entre as palavraschave: Supply chain manager skills com Competitiveness, Supply chain manager skills com competitive advantage, Supply chain manager competences com Competitiveness e Supply chain manager competences com competitive advantage, na base da EbscoHost (176 do total de 231), deu-se em função de que, dentre as cinco bases de pesquisa, ela é a que possui maior quantidade de títulos (1.104) relacionados à negócios.

Esses dados apontam que há correspondência entre competências/habilidades do gestor da cadeia de suprimentos e a competitividade/vantagem competitiva das empresas. A literatura consultada demonstrou a existência desse vínculo (seção 2.3), o qual permite identificar que as competências necessárias à gestão da cadeia de suprimentos podem ser divididas em duas dimensões: (i) competências técnicas (conhecimento das necessidades dos clientes e de como atendê-las, dos custos operacionais e financeiros, a integração interna da empresa e com fornecedores e a resolução dos problemas da cadeia de suprimentos) e habilidades comportamentais (saber trabalhar em equipe, ter boa comunicação e buscar focar no atendimento do cliente); (ii) conhecimento dos processos centrais da cadeia de suprimentos (fornecedores, empresa, clientes) e da concorrência (CLOSS; MOLLENKOPF, 2004; GIUNIPERO; HANDFIELD; ELTANTAWY, 2006; PRAJOGO; SOHAL, 2013).

Já o trabalho de Prajogo e Sohal (2013), um estudo empírico e que se baseia em um caso real, se destaca por se tratar de uma pesquisa realizada com gestores de cadeias de suprimentos na Austrália, buscando identificar as competências mais importantes para uma eficiente gestão dessa cadeia. Os respondentes apontaram que: (i) a capacidade de trabalhar de forma eficaz com pessoas e com equipes (transculturalmente, intra e interorganização); (ii) a capacidade de gerenciar relacionamentos em diversos contextos (transculturalmente, intra e interorganização); (iii) a capacidade de gerenciar riscos na cadeia de suprimentos e os problemas associados; (iv) a capacidade de fazer uso de técnicas numéricas para a tomada de decisão (por exemplo, forecasting e scheduling); (v) as habilidades de gerenciamento de projetos; e (vi) capacidade de liderar grandes projetos, foram as competências mais importantes, obtendo as médias de 4,39, 4,23, 4,11, 4,10, 4,09 e 4,0, respectivamente.

Embora tenha sido abordada a relação entre cadeias de suprimentos e competitividade, não foram encontrados no referencial teórico estudos que relacionassem a cadeia de suprimentos com a questão ambiental, no sentido de que a competitividade da empresa no contexto da cadeia de suprimentos envolve também o cuidado com os impactos ambientais decorrentes do funcionamento dessa cadeia. Outra lacuna observada foi a inexistência de pesquisas propondo um novo modelo de cadeia de suprimentos no contexto da Economia Circular. 


\section{Conclusão}

A concorrência globalizada fomenta constantes desafios às organizações, de modo que se torna capital que elas desenvolvam suas vantagens competitivas em um contexto de cadeia de suprimentos. Fazer uma eficiente gestão dessa cadeia de suprimentos é estratégico para a competitividade das empresas, no aspecto de atender aos clientes (e ocasionar neles a satisfação), obter os resultados planejados e se diferenciar estrategicamente dos concorrentes. O objetivo desta pesquisa foi o de analisar a importância das competências do gestor da cadeia de suprimentos para a competitividade das empresas. Com base na análise dos resultados apresentados, constata-se que a vantagem competitiva das empresas é plenamente efetivada quando estão inseridas em um ambiente integrado com fornecedores, clientes e/ou consumidores, sendo essa cadeia de suprimentos gerida com eficiência.

Para tal integração, os gestores da GCS necessitam ter a qualificação profissional adequada, possuindo (e desenvolvendo) competências, habilidades técnicas e conhecimentos que são requeridos, de modo que a operacionalização dessa cadeia fortaleça a competitividade da organização, para atender prontamente às necessidades dos clientes, gerar lucro e se diferenciar da concorrência. O perfil do gestor da GCS precisa abranger as competências técnicas (conhecimento das necessidades dos clientes e de como atendê-las, dos custos operacionais e financeiros, a integração interna da empresa e com fornecedores e a resolução dos problemas da cadeia de suprimentos), habilidades comportamentais (saber trabalhar em equipe, ter boa comunicação e foco no atendimento do cliente), conhecimento da concorrência e dos processos centrais da cadeia de suprimentos.

Quanto às limitações desta pesquisa, a principal é o fato de não ter discorrido acerca de quais competências necessárias para o gestor da GCS são natas e quais são as possíveis de serem aprendidas e desenvolvidas, bem como não fazer a distinção nem proporcionar o desenvolvimento e a medição das competências. Diante disto, sugere-se, como pesquisas futuras, estudos que identifiquem as competênciaschave para um gestor de cadeia de suprimentos que são natas e as que podem ser aprendidas, bem como as condições para desenvolvê-las. Outra proposta que surge é um estudo de casos reais de profissionais bem-sucedidos de GCS, em diferentes culturas, atividades de negócios e tamanhos de empresa, o que leva a questionamentos, tais como: quais são as competências que podem ser identificadas e aprendidas? Quais os resultados que o trabalho deles tem produzido fruto dessas competências (satisfação dos clientes, lucro, redução de custos e do índice de ruptura, ampliação do marketing share, entre outros)?

\section{Referências}

AB TALIB, M. S.; ABDUL HAMID, A. B. Application of critical success factors in supply chain management. International Journal of Supply Chain Management, [s.I.], v. 3, n. 1, Mar. 2014.

ABREU, A.; ALCANTARA, R. L. C. A gestão de pessoas e a estrutura organizacional sob a perspectiva da cadeia de suprimentos. REUNA, Belo Horizonte, v. 19, n. 1, p. 67-88, Jan.-Mar. 2014.

ABREU, A.; ALCANTARA, R. L. C. Entendendo a gestão de recursos humanos em cadeias de suprimentos: levantamento com o uso de revisão sistemática da literatura. Revista de Administração da UNIMEP (RAU), Piracicaba - SP, v. 12, n. 1, p. 100-128, Jan./Abr. 2014b.

BALLOU, R. H. Gerenciamento da cadeia de suprimentos: logística empresarial.5. ed. Porto Alegre: Bookman, 2006.

BATEMAN, T. S.; SNELL, S. A. Administração: novo cenário competitivo. 2. ed. São Paulo: Atlas, 2006.

BRAVO, M. I. R.; MORENO, A. R.; LLORENS-MONTES, F. J. Supply network-enabled innovations. An analysis based on dependence and complementarity of capabilities. Supply Chain Management: An International Journal, [s.I.], v. 21, n. 5, p. 642 - 660, 2016.

BREITBARTH, T.; HARRIS, P.; AITKEN, R. Corporate social responsibility in the European Union: a new trade barrier? Journal of Public Affairs, [s.I.], v. 9, n. 4, p. 239-255, Nov. 2009. 
CERTO, S. C.; PETER, J. P. Administração estratégica: planejamento e implantação de estratégias. 3. ed. São Paulo: Pearson, 2010.

CHANG, Cheng-Wen; CHIANG, D. M; PAI, Fan-Yun. Cooperative strategy in supply chain networks. Industrial Marketing Management, [s.I.], v. 41, p. 1114-1124, Oct. 2012.

CHOPRA, S.; MEINDL, P. Gerenciamento da cadeia de suprimentos. São Paulo: Prentice Hall, 2003.

CLOSS, D. J.; MOLLENKOPF, D. A. A global supply chain framework. Industrial Marketing Management, [s.I.], v. 33, n. 1, p. 37-44, Jan. 2004.

COOPER, M.C., LAMBERT, D.M.; PAGH, J.D. Supply chain management - more than a new name for logistics. International Journal of Logistics Management, [s.I.], v. 8, n. 1, p. 1-13, 1997.

FERREIRA, M. A bibliometric study on Ghoshal's managing across borders. Multinational Business Review, [s.I.], v. 19, n. 4, p. 357-375, 2011.

RODRIGUES FILHO, L. N. R.; AMIGO, R. J. R. Determinante da competitividade em mercados industriais. Revista de Administração, São Paulo, v. 35, n. 1, p. 23-31, jan./mar. 2000.

FLYNN, B. B.; HUO, B.; ZHAO, X. The impact of supply chain integration on performance: a contingency and configuration approach, Journal of Operations Management, [s.I.], v. 28, n. 1, p. 58-71, Jan. 2010.

FLEURY, M. T. L.; FLEURY, A. Construindo o conceito de competência. Revista de Administração Contemporânea, Curitiba, Edição Especial, p. 183-196, 2001.

GAMMELGAARD, B.; LARSON, P. D. Logistics skills and competencies for supply chain management. Journal of Business Logistics, [s.I.], v. 22, n. 2, p. 27-50, Sep. 2001.

GIUNIPERO, L. C., HANDFIELD, R. B.; ELTANTAWY, R. Supply management's evolution: key skill sets for the supply manager of the future. International Journal of Operations \& Production Management, [s.l.], v. 26, n. 7, p. 822-844, 2006.

GUERREIRO, R.; BIO, S. R.; MENDEL, S. F. Logística integrada, gestão da cadeia de suprimentos mensuração de custos e resultados logísticos: um estudo com empresas brasileiras. Advances in Scientific and Applied Accounting, São Paulo, v. 4, n. 1, p. 73-100, jan./abr. 2011.

GUNASEKARAN, A.; PATEL, C.; MCGAUGHEY, R. E. A framework for supply chain performance measurement. International Journal of Production Economics,[s.I.], v. 87, p. 333-347, Fev. 2004.

HARZING, A.; ALAKANGAS, S. Google Scholar, Scopus and the Web of Science: a longitudinal and cross-disciplinary comparison. Scientometrics,[s.I.], v. 106, n. 2, p. 787-804, Fev. 2016.

HITT, M. A.; MILLER, C. C.; COLELLA, A. Comportamento organizacional: uma abordagem estratégica. Rio de Janeiro: LTC, 2007.

INSTITUTO BRASILEIRO DE GEOGRAFIA E ESTATÍSTICA (IBGE). Estatísticas. Econômicas. Serviços. Demografia das Empresas. Disponível em: https://www.ibge.gov.br/estatisticas-novoportal/economicas/ servicos/9068-demografia-das-empresas.html?edicao=17053\&t=series-historicas. Acesso em: 29 out. 2017.

JACOMINO, G. P.; BÁNKUTI, S. M. S.; VIEIRA, F. D. G. Gestão da cadeia de suprimentos e marketing de relacionamento: proximidades e distanciamentos teóricos numa perspectiva integrada. Revista Eletrônica Científica do CRA-PR, Curitiba, v. 5, n. 1, p. 1-17, 2018.

LE BOTERF, G. Competénce et navigation professionnelle. Paris: Éditions d'Órganisation, 1999.

LOPES, L. C. et al. Práticas de contabilidade interorganizacional em indústrias de médio e grande porte instaladas na região metropolitana de Recife. Rev. Ciênc. Admin., Fortaleza, v. 20, n. 2, p. 664-691, jul./ dez. 2014. 
MENEGHETTI, F. K. O que é um ensaio teórico? Revista de Administração Contemporânea - RAC, Rio de Janeiro, v. 15, n. 2, p. 320-332, mar.-abr. 2011.

MENTZER, J. T. et al. Defining supply chain management. Journal of Business Logistics, [s.I.], v. 22, n. 2, p. 1-25, Autumn 2001.

MICHEL, M. H. Metodologia e pesquisa científica em ciências sociais. 3. ed. atual. e ampl. São Paulo: Atlas, 2015.

MIN, H.; ZHOU, G. Supply chain modeling: past, present and future. Computers \& Industrial Engineering, [s.I.], v. 43, n. 1-2, p. 231-249, Jul. 2002.

MONTEIRO, A. V.; MEDINA, E. A. M.; LAUREANO, F. O. A globalização e a necessidade de desenvolver competências para exportação. Revista de Negócios, Blumenau, v. 6, n. 2, p.33-40, abr./jun. 2001.

MOONEY, A. Core competence, distinctive competence, and competitive advantage: what is the difference?, Journal of Education for Business, [s.I.], v. 83, n. 2, p. 110-115, Aug. 2007.

MONTEZANO, L.; ABBAD, G. S.; FREITAS, P. F. P. Modelagem de competências profissionais de organização pública que atua no ramo de ciências forenses. In: EnANPAD - ENCONTRO CIENTíFICO DE ADMINISTRAÇÃO,40., 2016. Costa do Sauípe - BA. Anais [...]. Costa do Sauípe - BA: ANPAD, 2016. v. 1. p. 1-22.

NEUTZLING, D. M.; DA SILVA, M. E. A sustentabilidade em cadeias de suprimento a partir da visão de recursos e capacidades. Rev. Ciênc. Admin., Fortaleza, v. 22, n. 1, p. 42-71, jan./jun. 2016.

OLIVEIRA, W.; EL-AOUAR, W. A.; NÓBREGA, K. C. A elaboração de cenários estratégicos como vantagem competitiva. RAUnP - Revista Eletrônica do Mestrado Profissional em Administração da Universidade Potiguar, Natal, v. 10, n. 1, p. 41-58, jun./nov. 2017.

OLIVEIRA, W. F. M. et. al. Da Teoria Clássica à Contingencial: Contribuições à Competitividade das Organizações. RAUnP - Revista Eletrônica do Mestrado Profissional em Administração da Universidade Potiguar, Natal, v. 7, n. 2, p. 43-58, fev./maio 2015.

OLIVEIRA, W. F. M.; EL-AOUAR, W. A.; LEONE, R. J. G. Caso Rápido \& Barato: Vamos Entregar Logo a Encomenda?. REAd. Revista Eletrônica de Administração, Porto Alegre, v. 23, n. N. Especial, p. 394411, Dez. 2017.

ORLICKAS, E. Modelos de gestão: das teorias da administração à gestão estratégica. 2. ed. rev. atual. e ampl. Curitiba: Ibpex, 2011.

PAYÉS, M. A. M.; SILVA, O. R.; TEIXEIRA, R. D. Inovação, conhecimento e competitividade. In: SIMPÓSIO DE GESTÃO DA INOVAÇÃO TECNOLÓGICA, 25., 2008, Brasília. Anais [...]. Brasília - DF: ANPAD, 2008. p.1-10.

PIMENTEL, A. O método da análise documental: seu uso numa pesquisa histórica. Cadernos de Pesquisa, São Paulo, n.114, p. 179-195, nov. 2001.

PORTER, M. E. Estratégia competitiva: técnicas para análise de indústrias e da concorrência. 2. ed. Rio de Janeiro: Elsevier, 2004.

POSSAS, S. Concorrência e competitividade: notas sobre estratégia e dinâmica seletiva na economia capitalista. São Paulo: Hucitec, 1999.

PRAHALAD, C. K.; HAMEL, G. Competindo pelo futuro: estratégias inovadoras para obter o controle do seu setor e criar os mercados de amanhã. 24. ed. Rio de Janeiro: Elsevier, 2005.

PRAJOGO, D.; SOHAL, A. Supply chain professionals: a study of competencies, use of technologies, and future challenges. International Journal of Operations \& Production Management, [s. I.], v. 33, n. 11-12, p. 1532-1554, 2013.

RAMALHO, P. J. P.; SOUSA, A. J. Globalização e estratégias para o sector vitivinícola brasileiro: o polo 
emergente do Vale do São Francisco. RIAE - Revista Ibero-Americana de Estratégia, [s. I.], v. 8, n. 1, p. 05-27, Aug. 2009.

RANDONS, D. L. et. al. Uma síntese das publicações sobre competitividade: um levantamento dos principais tópicos vinculados ao tema. Rev. Ciênc. Admin., Fortaleza, v. 18, n. 2, p. 878-904, jul./dez. 2012.

REVISTA FORTUNE. Disponível em:<http://fortune.com/global500>. Acesso em 27 out. 2017.

RIBEIRO, J. S. A. N.; REISNETO, M. T. Gestão do desempenho como ferramenta para vantagem competitiva sustentável. Desafio Online, Mato Grosso do Sul, v.5, n.2, p. 261-275, Maio/Ago. 2017.

ROSSETTI, C. L.; DOOLEY, K. J. Job types in the supply chain management profession.

Journal of Supply Chain Management, [s. I.], v. 46, n. 3, p. 40-56, 2010.

SAMPIERI, R. H.; COLLADO, C. F.; LUCIO, P. B. Metodologia de pesquisa. 5. ed. São Paulo: Penso, 2013.

SAUBER, M. H.; MCSURELY, H. B.; TUMMALA, V.M. Rao. Developing supply chain management program: a competency model. Quality Assurance in Education, [s. I.], v. 16, n. 4, p. 375-391, Jul. 2008.

SCAVARDA, L. F.; SCAVARDA, A. J. Viabilizadores para a supply chain management. FACES R. Adm, Belo Horizonte, v. 3, n. 2, p. 11-25, jul./dez. 2004.

SCHREIBER, D.; VILELA JÚNIOR, D. C.; BANDEIRA, D. L. Estudo de competitividade de redes de pequenas empresas da região do Vale dos Sinos. In: ENCONTRO DA ANPAD (EnANPAD), 31., 2007, Rio de Janeiro. Anais [...]. Rio de Janeiro: ANPAD, 2007. p. 1-16.

SILVA, J. C.; FECHIO, A. C.; PEPECE, O. M. C. O ato de presentear: uma revisão das publicações entre os anos de 2000 e 2013. ESTUDO \& DEBATE, Lajeado, v. 21, n. 2, p. 180-200, 2014.

SNELL, S.; BOHLANDER, G. Administração de recursos humanos. São Paulo: Cengage Learning. 2013.

SNYDER, C. A case study of a case study: analysis of a robust qualitative research methodology. The Qualitative Report, [s. I.], v. 17, p. 1-21, 2012.

STARIK, M.; KANASHIRO, P. Toward a Theory of Sustainability Management: Uncovering and Integrating the Nearly Obvious. Organization \& Environment, [s. I.], v. 26, n. 1, p. 7-30, 2013.

STOCK, J. R.; BOYER, S. L. Developing a consensus definition of supply chain management: a qualitative study. International Journal of Physical Distribution \& Logistics Management, [s. I.], v. 39, n. 8, p. 690-711, 2009.

TAN, K.; LYMAN, S. B.; WISNER, J. D. Supply chain management: a strategic perspective. International Journal of Operations \& Production Management, [s. I.], v. 22, n. 6, p. 614-631, 2002.

TRANFIELD, D.; DENYER, D.; SMART, P. Towards a methodology for developing evidence-informed management knowledge by means of systematic review. British Journal of Management, [s. I.], v. 14, p. 207-222, 2003.

VAN DER HEIJDEN, K. Planejamento por Cenários: a arte da conversação estratégica. Porto Alegre: Bookman, 2009.

VAN HOEK, R.I.; CHATHAM, R; WILDING, R. Managers in supply chain management: the critical dimension. Supply Chain Management: An International Journal, [s. I.], v. 7, n. 3, p. 119-125, 2002.

WAGNER III, J. A.; HOLLENBECK, J. R. Comportamento organizacional: criando vantagem competitiva. 2. ed. São Paulo: Saraiva, 2009. 
YEN-TSANG, C.; CSILLAG, J. M.; SIEGLER, J. Theory of reasoned action for continuous improvement capabilities: a behavioral approach. Revista de Administração de Empresa - RAE, São Paulo, v. 52, n. 5, p. 546-564, Sept./Oct. 2012.

Submetido em: 09/11/2017

Aceito em: 05/04/2019 